\title{
Performance Profile of Online Training Assessment Based on Virtual Reality:
} Embedded System versus PC-only

\author{
José Taunaí Segundo, Elaine Soares, Liliane S. Machado, and Ronei M. Moraes* \\ Federal University of Paraíba, João Pessoa/PB, Brazil \\ \{taunai2, elaineanita1\}@gmail.com, liliane@di.ufpb.br, \\ ronei@de.ufpb.br
}

\begin{abstract}
Training systems based on virtual reality are used in several areas of human activities. In some kinds of training is important to know the trainee's skills. It can be done in those systems but requires high-end computers to achieve good performance. Recently, the use of embedded systems connected to the training system was proposed for training assessment, with the goal of decreasing requirements of the main system. However, some questions are still open and a deep study of this proposal was not performed. This paper provides answers for some of those questions.
\end{abstract}

Keywords: Embedded Systems, Virtual Reality, Training Assessment, Fuzzy Naive Bayes, Possibility and Necessity Measures.

\section{Introduction}

Continuous learning and improvement of skills for staffs are a demand of several areas to guarantee good offer of services. With this purpose, applications based on virtual reality (VR) have been developed in order to provide realistic training, particularly in the medical area [3]. In those systems, users are exposed to simulated problems in 3D environments to practice and get technological and psychological skills to perform them in a real condition. Also, VR systems demand integration and synchronizations of routines, hardware and techniques [1], what requires high processing rates to provide real time feedback.

One of the main advantages of training in VR simulators is the possibility of monitoring user actions to register their movements. Then, information as force, position and acceleration, among others performed with interaction devices (as haptics) must be acquired and processed during all the training session. This information is used to feed assessment routines that must provide feedback about users skills. Thus, an online feedback [3] will demand expressive time of CPU, which can compromise the other tasks of the simulator. Moraes and Machado [2] proposed an architecture for assessment based on embedded systems. In this architecture, an embedded system is connected to the VR simulator to enhance the execution of the

\footnotetext{
* Corresponding author.
} 
assessment tasks and release the CPU for other tasks related to the simulation. However, no further studies to analyze the efficiency of the architecture, its limitations to provide online assessment and its performance if compared to a CPU based approach were identified in the literature. The paper has as goal to provide this analysis for two previously proposed online methods based on fuzzy sets: Fuzzy Naive Bayes (FNB) [5] and Possibility and Necessity Measures (PMN) [14]. Both were implemented in a CPU and also in an embedded system. Monte Carlo simulations were used to describe profiles for both methods with increasing size databases.

\section{Virtual Reality and Training Assessment}

VR for training of procedures allows simulating real problems in a realistic way, avoiding risks and ethical issues [3], as the acquisition of guinea pigs or cadaveric bodies. Advantages of the use of VR for training are related to the variability of cases that can be simulated, including rare and atypical occurrences, the possibility of repetition without degradation of materials and the absence of risks for the people involved.

The use of special devices in VR systems allows reaching high levels of immersion and interactivity, providing for users the feeling of presence by the manipulation of elements in the virtual environment [8]. Those devices usually explore users's senses as the sight, hearing and touch. The VR system processes all interactions and the feedback to be provided. Also, the VR system is responsible by the synchronization of all tasks in order to guarantee the sequence and coherence among the several tasks. These tasks include the calculus of physical phenomena, lighting and collision detection, as examples. Because interaction devices can acquire data with rates that start on $30 \mathrm{~Hz}$, the amount of information processed in a simulation can be massive, depending on the type of interaction. Haptic devices, as example, can capture interaction in rates between 500 and $1000 \mathrm{~Hz}$.

Since VR systems are computational simulations, interaction data of the procedure can be collected and used to assess trainees' performance. This can occur in two different ways: offline and online. Offline assessment methods are those that cannot provide immediate feedback for users: post-analysis of recorded training [10] sessions or questionnaires answered by users [11]. However, users can forget their actions after some time, which gives to this type of assessment a lower didactic impact. Online assessment methods can provide real-time feedback and trainees can immediately repeat the training and try to correct their actions to improve their performance. It is important since it can provide a more effective learning process. Several methods for online assessment have been proposed for medical simulators based on VR $[4,5,6,9,14]$.

The calibration of the evaluation system is necessary to acquire and label correct and incorrect ways to perform the procedure. It is provided by an expert of the simulator subject that executes several times the procedure in different ways in order to generate parameters for each execution. A previously defined number for classes of performance is used to label each execution. All interaction and environment parameters are acquired during this process to be used by the assessment method, which is normally based on a pattern recognition technique. As example, a $\mathrm{M}=3$ 
number of classes can refers to 1: "good performance“, 2 - "regular performance“, 3 "bad performance" [14].

\section{Assessment Methods and Decision Rule}

\subsection{Fuzzy Naive Bayes (FNB) Method}

A fuzzy set A in $\Omega$ is defined for each element $\omega \in \Omega$ as a mapping $\mu_{\mathrm{A}}$, called membership function, which can associate each element from $\Omega$ to $[0,1]$, and is interpreted as the degree of membership of $\omega$ in A [7]. Some fuzzy versions for the Naive Bayes classifier were proposed. In this work we follow the version proposed by Störr [17] and used by [5] as a kernel of an assessment system for training based on VR.

Formally, let be $\Omega=\{1, \ldots, M\}$ the classes of performance in space of decision, where $M$ is the total number of classes of performance. Let be $w_{i}, i \in \Omega$ the class of performance for a trainee. It is possible to determine the class of performance most probable for this trainee given a data vector $\boldsymbol{X}=\left\{X_{1}, X_{2}, \ldots, X_{n}\right\}$ and assuming that each $X_{k}, k=1, \ldots, n$ is a fuzzy variable, with normalized membership functions $\mu_{i}\left(X_{k}\right)$, where $i=1, \ldots, M$. The method is defined by [5]:

$$
P\left(w_{i} \backslash X\right)=(1 / S) P\left(w_{i}\right) * \mathrm{P}\left(\mathrm{W}_{\mathrm{i}}\right) \quad \prod^{\mathrm{n}}{ }_{\mathrm{k}=1} \quad\left[P\left(X_{k} \backslash w_{i}\right) \mu_{i}(X)\right], \mathrm{i} \in \Omega
$$

where $S$ is a scale factor which depends on $X_{1}, X_{2}, \ldots, X_{n}$.

The classification rule for Fuzzy Naive Bayes is: select performance class $w_{i}$ for the vector $\boldsymbol{X}$ if:

$$
P\left(w_{i} \backslash X\right)>P\left(w_{j} \backslash X\right) \text { for all } i \neq j \text { and } i, j \in \Omega
$$

\subsection{Possibility and Necessity Measures (PMN) Method}

Let A be a fuzzy subset of $\Omega$, with its membership function $\mu_{\mathrm{A}}$, and let $\mathrm{X}$ be a variable which assumes values $\omega$ in $\Omega$. Then, the possibility distribution $\pi$ is a function associated to $\mathrm{X}$ and is defined as:

$$
\pi_{\mathrm{X}}(\omega)=\mu_{\mathrm{A}}(\omega)
$$

The possibility measure $\Pi$ and the necessity measure are defined respectively by:

$$
\Pi(\mathrm{A})=\sup \{\pi(\mathrm{u}) \mid \mathrm{x} \in \mathrm{A}\} \quad \text { and } \quad \mathrm{N}(\mathrm{A})=\inf \{1-\pi(\mathrm{u}) \mid \mathrm{x} \notin \mathrm{A}\} .
$$

Other relations between them were provided in [16]:

$$
\begin{gathered}
\Pi(\varnothing)=\mathrm{N}(\varnothing)=0 ; \quad \Pi(\mathrm{A})=\mathrm{N}(\mathrm{A})=1 ; \\
\max (\Pi(\mathrm{A}), \Pi(\overline{\mathrm{A}}))=1 ; \min (\mathrm{N}(\mathrm{A}), \mathrm{N}(\overline{\mathrm{A}}))=0 ;
\end{gathered}
$$

The possibility and the necessity measures are dual:

$$
\Pi(\mathrm{A})=1-\mathrm{N}(\overline{\mathrm{A}}) \text { and } \mathrm{N}(\mathrm{A})=1-\Pi(\overline{\mathrm{A}}) .
$$


Some relations between them can be provided [16]:

$$
\Pi(\mathrm{A}) \geq \mathrm{N}(\mathrm{A}) ; \quad \mathrm{N}(\mathrm{A})>0 \Rightarrow \Pi(\mathrm{A})=1 ; \quad \Pi(\mathrm{A})<1 \Rightarrow \mathrm{N}(\mathrm{A})=0
$$

Let $A$ and $B$ be fuzzy subsets of $\Omega$, with membership functions $\mu_{A}$ and $\mu_{B}$, respectively. Let $X$ be a variable which assumes values $\omega \in \Omega$. The conditional possibility and necessity measures are given by [16]:

$$
\begin{array}{cc}
\Pi(\mathrm{A} \mid \mathrm{B})=\max _{\mathrm{u} \in \mathrm{X}} \quad \min \left(\mu_{\mathrm{A}}(\mathrm{u}), \pi_{\mathrm{B}}(\mathrm{u})\right) \text { and } \\
\mathrm{N}(\mathrm{A} \mid \mathrm{B})=\min _{\mathrm{u} \in \mathrm{X}} \quad \max \left(\mu_{\mathrm{A}}(\mathrm{u}), 1-\pi_{\mathrm{B}}(\mathrm{u})\right) .
\end{array}
$$

From equation (4) is possible to construct an interval for the real value of the class of performance $w_{i}$, given each feature $X_{k}$, with $k=\{1, \ldots, n\}$, from the training data $\boldsymbol{X}=$ $\left\{X_{1}, X_{2}, \ldots, X_{n}\right\}$ from a user [14]:

$$
\mu_{\omega \mathrm{i}}\left(X_{j}\right) \in\left[\mathrm{N}\left(\omega_{\mathrm{i}} \mid X_{k}\right) ; \Pi\left(\omega_{\mathrm{i}} \mid X_{k}\right)\right] .
$$

The domain of membership function for the class of performance $\omega_{\mathrm{i}}$ is an interval where the minimum value is the minimum compatibility and the maximum value is the maximum compatibility:

$$
\mu \omega_{\mathrm{i}}(\mathbf{X}) \in\left[\text { compat }_{\min } ; \text { compat }_{\max }\right]
$$

As the class of performance $w_{i}$ is expressed by a conjunction of features $X_{j}$, then this aggregation is performed by a t-norm. In this case, the "min" operator preserves the semantics of possibility and necessity measures [14]:

$$
\text { compat }_{\min }=\min _{\mathrm{k}}\left(\mathrm{N}\left(\omega_{\mathrm{i}} \mid X_{k}\right)\right) \quad \text { and } \quad \operatorname{compat}_{\max }=\min _{\mathrm{k}}\left(\Pi\left(\omega_{\mathrm{i}} \mid X_{k}\right)\right) .
$$

The defuzzification process can be done using, for instance, the centroid method, where $C\left[\mu_{\text {wi }}(\boldsymbol{X})\right]$ is the centroid between compat ${ }_{\min }$ and compat ${ }_{\max }$ for the pertinence function of class $w_{i}$, according to $\boldsymbol{X}$. Then, the decision rule is: select performance class $w_{i}$ for the vector $\boldsymbol{X}$ if [14]:

$$
w_{i}=\arg \max { }_{1 \leq i \leq \mathrm{M}} \mathrm{C}\left[\mu_{\mathrm{wi}}(\boldsymbol{X})\right]
$$

\section{$4 \quad$ Embedded System}

An embedded system is a combination of hardware and software with additional components (mechanical and/or electronic) performing a dedicated function [8]. Its hardware is specifically designed to fulfill requirements of a system making it cheaper. This kind of device is also characterized by having higher quality, higher reliability and lower cost components [8] than other computer systems. Its architecture is generally similar to that of a computer system and may be composed by main memory, secondary memory, processor and buses input and output, such as: USB port, VGA port, network adapter and others, according to the task to be performed. 
As mentioned in Section 2, a VR system can demand processing of massive data, which can overload the main system. To relief it, we take the advantage of using an embedded system to execute assessment tasks to meet timing requirements [3].

\section{$5 \quad$ Methodology}

The objective of this paper is analyze the efficiency of the architecture proposed by [2] in producing good results, investigating the limits of this architecture in providing online assessments and what are performance relationships between the assessments provide by a PC-only system and the architecture proposed by [2]. Initially, the authors developed computational programs for both assessment methods, a PC-only system and an embedded system, which configurations were, respectively: Athlon 64 X2 AMD processor, 2,4GHz, 2GB of DDR2 RAM, under Fedora Linux and Geode AMD processor, $500 \mathrm{MHz}, 256 \mathrm{MB}$ of DDR RAM, under Gentoo Linux. After, were used Monte Carlo simulations [15] with 2000 databases, where the smallest database contained three dimensional vectors with 1000 positions each, with four classes of performance, as showing in the Table 1. Databases with increasing sizes were randomly generated, starting from the smaller one, and the performance profiles were outlined for both methods. Performance classes were defined from the combination of random variables and those from Gaussian distributions generated with the predefined parameters, with at least $30 \%$ of intersection. Variables and parameters used are present in Table 2.

Table 1. Classes setup

\begin{tabular}{ll}
\hline Class & Variables \\
\hline I & ABC \\
II & ABD \\
III & ABE \\
IV & ABK \\
\hline
\end{tabular}

Table 2. Variables and parameters

\begin{tabular}{ll}
\hline Variable & Normal parameters \\
\hline A & $\mathrm{N}(0 ; 1)$ \\
B & $\mathrm{N}(-2 ; 1)$ \\
C & $\mathrm{N}(10 ; 20)$ \\
D & $\mathrm{N}(19 ; 20)$ \\
E & $\mathrm{N}(1 ; 20)$ \\
K & $\mathrm{N}(-15 ; 8)$ \\
\hline
\end{tabular}

The calibration phase used 10 databases with 1000 positions each one. To check the performance of the methods were used in the calibration phase between 100 and 2000 databases. Each database had the same number of positions, but they were used several times to increase the final database. So, the final database had 20000 positions to assess. In the case of the trainee assessment performed by PC-only, the method was executed locally. However, for the embedded system was necessary defining a framework for communication via Ethernet network (client-server via TCP/IP). The server application ran on the PC. It simulated the generation of data from the VR system, received and displayed the assessment report. The client application ran on embedded system and received data from the server, conducted the trainee's assessment, calculated its own average time of execution and sent the final report to the server. The average accuracy of each method was verified using the Kappa coefficient proposed by Cohen [12] and recommended by the literature of pattern recognition [13]. 


\section{$6 \quad$ Results and Discussions}

From the Monte Carlo simulation data, as described above, were obtained confusion matrices and the average time of execution for both methods, which are presented in Tables 3 for FNB method and in Table 4 for PNM method. The main diagonal of those matrices represents the correct classifications for each method. As was expected, the results obtained from the assessment system-only running on the PC and embedded system were numerically equal.

Using the Table 3 was computed the Kappa coefficient for FNB, which resulted in 0.653 with standard deviation 0.006 . The percentage of correct classification was $73.95 \%$. It were noticed 2084 misclassifications and those errors were concentrated in the classes I and IV. The percentage of correct classification for the class I was poor: only $1.55 \%$. However, FNB achieved good results for the classes II and III $(100.00 \%)$. The percentage was $94.25 \%$ for the class IV. The average time of execution for the FNB method was 0.2098 seconds for the embedded system and 0.0139 seconds for the PC-only.

For the PNM method, the time measured was 0.2104 and 0.0140 seconds for the embedded system and for the PC-only, respectively. From Table 4 data, the Kappa coefficient obtained was 0.693 with standard deviation 0.006 . The percentage of correct classification was $77.00 \%$. The number of misclassifications was 1840 , distributed in the classes I, II and III. The percentages for correct classifications were $94.10 \%, 20,10 \%$ and $96,80 \%$ for the classes I, II and III, respectively. IN this case, PNM achieved good results for the class IV (100.00\%). These results were not so good as those presented by [2], specially for the class II.

Table 3. Fuzzy Naive Bayes Error Matrix

Table 4. Possibility and Necessity Error Matrix

\begin{tabular}{|c|c|c|c|c|c|c|c|c|c|}
\hline Class & $I$ & $I I$ & $I I I$ & $I V$ & Class & $\bar{I}$ & $\overline{I I}$ & III & $I V$ \\
\hline I & 31 & 633 & 1336 & 0 & I & 1822 & 0 & 178 & 0 \\
\hline II & 0 & 2000 & 0 & 0 & II & 1589 & 402 & 9 & 0 \\
\hline III & 0 & 0 & 2000 & 0 & III & 64 & 0 & 1936 & 0 \\
\hline IV & 0 & 0 & 115 & 1885 & IV & 0 & 0 & 0 & 2000 \\
\hline
\end{tabular}

Both methods satisfied the requirement of processing time lower than one second either running in the embedded system as in the PC-only. However, the ratio between processing times when running in the PC-only and when running in the embedded system, with the configurations described above, was around 15 times. It was expected, and this is clearly due to the differences between their configurations.

It can be seen in the Figure 1 (left) that both curves are linear. The same can be observed for the PC-only (Figure 1 - right) when the databases are lower than 1500. After that, the behavior for PC-only simulations is nonlinear. This change of behavior can be due to the scheduling of the operational system. The time to run 1500 databases in the PC-only is approximately the same to run 100 databases in the embedded system. The graphic on left has a 400 seconds scale with 420 s. The graphic on right has a 40 seconds scale and the limit of curves are below this value. 
An embedded system was designed for this specific task but not for the PC-only. In this last one, its operational system spends time to perform other tasks not related to the assessment task or to VR-based simulation. It should be noted also that, although the total time for carrying out the evaluation for all databases was higher than one second (see Figure 1), the ratio for the amount of total time positions of each vector remained below one second to any number of databases and for both methods.
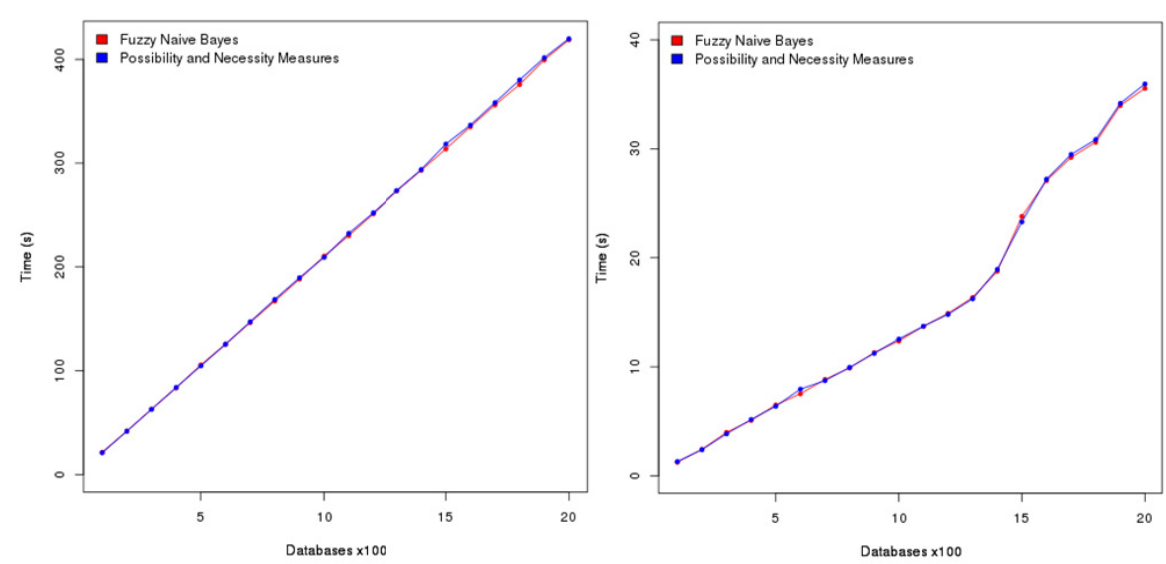

Fig. 1. Processing times for embedded system (left) and for the PC-only (right)

\section{Conclusions}

An architecture for assessment based on embedded systems was proposed by [2] some years ago. In the present paper were presented answers for some questions open, as the real efficiency of this architecture, its limitations to provide online assessment and its performance if compared to a CPU based approach. The results obtained shown this assessment architecture can provide the same results as methods implemented for PC-only. When comparing the processing times to perform the assessment task using the embedded system, the PC-only was around 10.5 times faster, but the embedded system also achieved the requirement of assessment time lower than 1 second.

By the results obtained was possible to confirm the proposal [2] of transferring assessment tasks to an embedded system and leave the main system available to process the tasks related to the VR simulation. It was important to observe that even with a less powerful configuration, the embedded system was able to provide fast answers. Therefore, the use of assessment methodologies, even if computationally expensive, can be considered to be implemented in embedded systems without compromising the simulation performance. 
Acknowledgement. This work is supported by the National Council for Scientific and Technological Development (CNPq): processes 310470/2012-9, 310561/2012-4 , PIBIC//UFPB and 181813/2010-6 (INCT-MACC).

\section{References}

1. Machado, L.S., Mello, A.N., Lopes, R.D., Odone Fo, V., Zuffo, M.K.: A Virtual Reality Simulator for Bone Marrow Harvest for Pediatric Transplant. Studies In Health Technology and Informatics 81, 293-297 (2001)

2. Moraes, R.M., Machado, L.S.: Using Embedded Systems to Improve Performance of Assessment in Virtual Reality Training Environments. In: Int. Conf. Engineering and Technology Education (Intertech 2008), Santos, Brazil, pp. 140-144 (2008)

3. Moraes, R., Machado, L.: Development of a Medical Training System with Integration of Users' Assessment. In: Kim, J.-J. (ed.) Virtual Reality, ch. 15. Intech (2011)

4. Moraes, R.M., Machado, L.S.: Assessment Based on Naive Bayes for Training Based on Virtual Reality. In: Int. Conf. Engineering and Computer Education (ICECE 2007), Santos, Brasil, pp. 269-273 (2007)

5. Moraes, R.M., Machado, L.S.: Another Approach for Fuzzy Naive Bayes Applied on Online Training Assessment in Virtual Reality Simulators. In: Safety, Health and Environmental World Congress (SHEWC 2009), Mongaguá, Brazil, pp. 62-66 (2009)

6. Moraes, R.M., Machado, L.S.: Online Assessment in Medical Simulators Based on Virtual Reality Using Fuzzy Gaussian Naive Bayes. Journal of Multiple-Valued Logic and Soft Computing 18(5), 479-492 (2012)

7. Zadeh, L.A.: Fuzzy Sets. Information and Control 8, 338-353 (1965)

8. Barr, M.: Programming Embedded Systems in C and C++. O'Reilly (1999)

9. Moraes, R.M., Machado, L.S.: Gaussian Naive Bayes for Online Training Assessment in Virtual Reality-Based Simulator. Mathware \& Soft Computing 16, 123-132 (2009)

10. McBeth, P.B., et al.: Quantitative Methodology of Evaluating Surgeon Performance in Laparoscopic Surgery". Studies in Health Technology and Informatics 85, 280-286 (2002)

11. Dinsmore, M., Lagrana, N., Burdea, G., Ladeji, J.: Virtual Reality Training Simulation for Palpation of Subsurface Tumors. In: IEEE VRAIS 1997, pp. 54-60. IEEE Press (1997)

12. Cohen, J.: A coefficient of agreement for nominal scales. Educational and Psychological Measurement 20, 37-46 (1960)

13. Webb, A.: Statistical Pattern Recognition, 2nd edn. John Wiley, Chichester (2005)

14. Machado, L.S., Moraes, R.M.: Medical Skills Assessment in Training Based on Virtual Reality Using a Possibilistic Approach. In: 10th Int. FLINS Conf (FLINS 2012), Istanbul, Turkish, pp. 339-345 (2012)

15. Gentle, J.E.: Random Number Generation and Monte Carlo Methods, 2nd edn. Springer Science (2005)

16. Dubois, D., Prade, H.: Possibility theory. Plenum Press, New-York (1988)

17. Störr, H.-P.: A compact fuzzy extension of the naive Bayesian classification algorithm. In: 3rd International Conference on Intelligent Technologies and Vietnam-Japan Symposium on Fuzzy Systems and Applications, Hanoi, Vietnam, pp. 172-177 (2002) 